\title{
Catalytic Asymmetric Alkylations via Phase-Transfer Catalysis
}

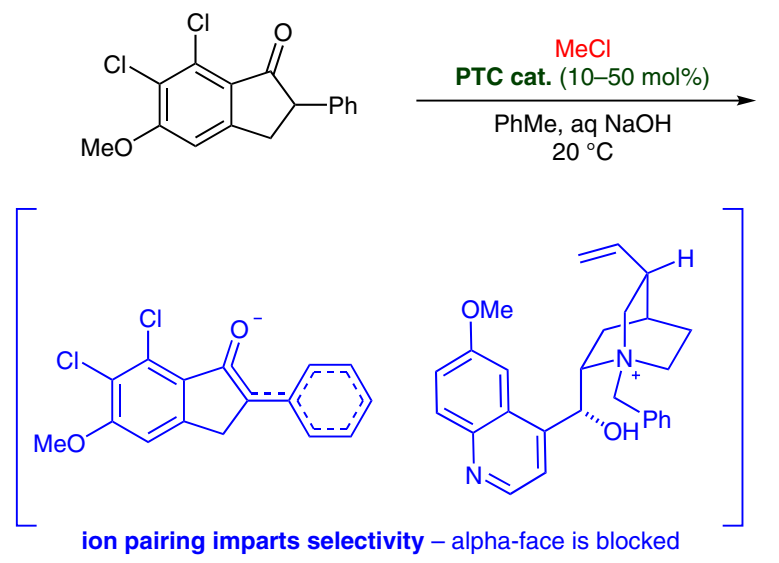

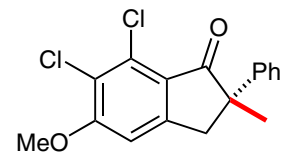

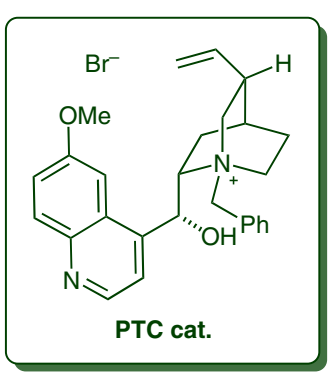

\section{Category}

Organo- and Biocatalysis

\section{Key words}

phase-transfer catalysis

alkylation

indanone anions

benzylcinchoninium cations
Significance: Efficient and facile asymmetric alkylation reactions were a long-sought objective in organic synthesis prior to this report. Most reported literature precedents at the time involved several steps and required the use of stoichiometric quantities of chiral auxiliaries. The authors of this work reported what became the first efficient catalytic and enantioselective alkylation in the asymmetric synthesis of (+)-indacrinone through chiral phase-transfer catalysis. On the basis of space-filling molecular models, the authors surmised that ion pairing between the indanone anion and benzylcinchoninium cation effectively blocks the alpha-face of the substrate, thereby giving rise to the observed enantioselectivity of the reaction.
Comment: This reaction marked the beginning of modern asymmetric phase-transfer catalysis with enantiopure quaternary ammonium salts. The development of this reaction showed that each of the reaction parameters was critical to the selectivity and success of the chiral phase-transfer catalysis approach. In general, nonpolar solvents, along with increased dilution of the reaction mixture, lower temperatures, decreased alkyl halide concentrations, and increased $\mathrm{NaOH}$ concentrations of up to $50 \%$ produced higher enantiomeric excesses. The catalyst concentration affected the rate of the reaction, but had only a weak effect on the enantioselectivity. Interestingly, adding substituents of increasing electron-withdrawing power onto the catalyst also improved the catalyst selectivity, presumably by enhancing the $\pi$-interaction between the catalyst cation and the substrate anion. 